\title{
Antibiotics-impregnated ventricular catheter versus systemic antibiotics for prevention of nosocomial CSF and non-CSF infections: a prospective randomised clinical trial
}

\author{
George K C Wong, ${ }^{1}$ Margaret Ip, ${ }^{2}$ Wai S Poon, ${ }^{1}$ Christopher W K Mak, \\ Rebecca $Y \mathrm{~T} \mathrm{Ng}^{1}$
}

${ }^{1}$ Division of Neurosurgery, Prince of Wales Hospital, The Chinese University of Hong Kong, Hong Kong, China ${ }^{2}$ Department of Microbiology, Prince of Wales Hospital, The Chinese University of Hong Kong, Hong Kong, China

\section{Correspondence to} Dr G K C Wong, Division of Neurosurgery, Department of Surgery, Prince of Wales Hospital, Shatin, New Territories, Hong Kong 852, China; georgewong@surgery. cuhk.edu.hk

Received 26 October 2009 Revised 12 January 2010 Accepted 14 January 2010 Published Online First 12 May 2010

\section{UNLOCKA}

This paper is freely available online under the BMJ Journals unlocked scheme, see http:// innp.bmi.com/site/about/ unlocked.xhtml

\begin{abstract}
Background In recent years, cranial ventricular catheters impregnated with antimicrobial agents have become available. Theoretically, they provide antibiotic prophylaxis locally without the associated complications of opportunistic nosocomial infections. This study aims to compare antibiotic impregnated catheters with conventional catheters coupled with systemic antibiotics. Methods Patients undergoing emergency neurosurgical operations were recruited. Patients were randomly assigned to antibiotic impregnated catheters (Bactiseal, Codman, Johnson \& Johnson, Raynham, MA, USA) or conventional catheters coupled with systemic antibiotics. Results 184 neurosurgical patients were enrolled between April 2004 and December 2008. Mean duration of ventricular catheter was 10 days for both groups. The proportion of patients with nosocomial infection was not significantly different: $57 \%(51 / 90)$ in the Bactiseal group and $51 \%(48 / 94)$ in the conventional group (OR 1.3, 0.7 to 2.2). There were also no differences in secondary outcome measures (CSF infection, intensive care unit stay, acute hospital stay and functional outcome) between the two groups.

Conclusions Antibiotic impregnated catheters are as effective as systemic antibiotics in the prevention of CSF infection and their corresponding nosocomial infection rates are not significantly different.

The study is registered at http://www.ClinicalTrials.gov (NCT00286104).
\end{abstract}

\section{INTRODUCTION}

External ventricular catheters are used for intracranial pressure monitoring and temporary CSF drainage in neurosurgery. An incidence of ventriculostomy related CSF infection has been quoted as between $2 \%$ and $23 \%$ in the literature. ${ }^{1-7}$ In recent years, CSF shunt catheters impregnated with antimicrobial agents have become available. One antibiotic impregnated catheter (Bactiseal, Codman, Johnson \& Johnson, Raynham, MA, USA) is a silicone catheter impregnated with $0.15 \%$ clindamycin and $0.054 \%$ rifampicin. Experimental study had shown that it provides protection against Staphylococcus aureus and coagulase negative staphylococci strains for between 42 days and 56 days. $^{8}$ Theoretically, it provides antibiotic prophylaxis locally without associated complications of opportunistic nosocomial infections. This beneficial effect had been shown to reduce positive CSF cultures but no data in terms of outcome and nosocomial infection are available in the literature. ${ }^{9}$ A previous prospective study in our unit showed that the use of dual antibiotics prophylaxis in patients with external ventricular drainage was associated with a decreased incidence of CSF infection but was complicated by opportunistic extracranial infection. ${ }^{10}$ The current practice in our unit is to cover with prophylactic dual antibiotics unless guided by microbiology results for all patients with external ventricular drains. ${ }^{11}$ This provided a unique opportunity to compare the effectiveness of antibiotic impregnated catheters with systemic antibiotics in terms of nosocomial infection and CSF infection.

\section{METHODS}

This was an investigator initiated, open labelled, randomised controlled trial performed at a university affiliated neurosurgical centre in Hong Kong (Prince of Wales Hospital, Chinese University of Hong Kong) between 1 April 2004 and 31 December 2008. The study protocol received ethics committee approval from the participating centre. All participants or their legally acceptable representatives provided written informed consent.

\section{Participants}

Patients who had clinical indications for external ventricular drain insertion and aged 18 years or above, or their next of kin, were approached for participation in the trial. Exclusion criteria included known intracranial or extracranial infections, allergy to rifampicin, clindamycin, ampicillin/sulbactam, ceftriaxone or silicone, and pregnancy.

\section{Procedures}

All patients were treated according to a standard protocol in the neurosurgical high dependency unit or intensive care unit. Patients were randomised into one of the two groups: (1) Bactiseal group: periprocedural antibiotics only-that is, intravenous ampicillin-sulbactam and ceftriaxone and insertion of the antibiotic impregnated $(0.15 \%$ clindamycin and $0.05 \%$ rifampicin) ventricular catheter (Bactiseal, Codman, Johnson and Johnson; (2) Conventional group: periprocedural antibiotics and prophylactic dual antibiotics - that is, intravenous ampicillin-sulbactam and ceftriaxone, and insertion of ventricular catheter without impregnation of antibiotics. 'Prophylactic' means the use of the 
antibiotics listed above, as long as the ventricular catheter is in situ, unless change is necessary clinically due to concurrent infection. This was an open labelled study with the Bactiseal catheter coloured orange and plain ventricular catheters coloured white, as commercially available. The ventricular catheter could be inserted as a separate procedure or in the setting of craniotomy, as determined clinically. We performed all our burr holes and ventricular drain insertions under stringent aseptic conditions in the operating theatre with a subgaleal tunnelling of around $5 \mathrm{~cm}$. Dressing were applied to cover the wound. Standard protocols were implemented for care of ventricular catheter to avoid bacterial contamination of the system. CSF was collected using an aseptic technique from the three way connector just distal to the ventricular catheter every 5 days or on evidence of clinical sepsis. Definition of CSF infection was positive CSF bacterial culture together with CSF white cell count $>10 / \mathrm{mm}^{3}$, CSF protein level $>0.8 \mathrm{~g} / \mathrm{l}$ and CSF to serum glucose ratio $<0.4$. We did not perform regular exchange of external ventricular drain according to the published randomised controlled clinical trial of our unit. ${ }^{12}$

\section{Statistical analysis}

Data management and statistical analysis were done by the research team of the Division of Neurosurgery, Prince of Wales Hospital, Chinese University of Hong Kong, Hong Kong. For sample size estimation, ${ }^{13}$ we used a difference of nosocomial infection rates between $40 \%$ and $20 \%,{ }^{10}$ with $5 \%$ level of significance and $80 \%$ power. A total of 180 patients with $(90$ patients in each arm) were calculated as the target sample size. Analyses were done on an intention to treat basis.

Primary outcome was acquisition of nosocomial infection of all sites, including the chest, urinary tract, bacteraemia and of the CNS, as confirmed by positive culture. Secondary outcome included CNS infection, ICU stay, acute hospital stay and functional outcome at 6 months. The follow-up period for determination of these infections was the first 3 months of insertion of the intraventricular catheterisation or duration of hospitalisation, whichever was shorter. Glasgow Outcome Score Extended ${ }^{14}$ was stratified as unfavourable (score $\leq 4$ ) or favourable outcome (score 5-8). The associations (nosocomial infection, CNS infection and functional outcome) with the two groups were determined using $\chi^{2}$ tests or Fisher's exact tests as appropriate. ORs with $95 \%$ CIs were calculated. The Mann-Whitney U tests were used to assess the other quantitative secondary outcome (ICU stay and acute hospital stay), and mean differences between treatment groups were presented with 95\% CIs. Probabilities of no CNS infection between treatment groups were compared using the log rank test.

The trial data were collected on printed forms and subsequently entered on to a computer by use of ACCESS 2003 software (Microsoft Inc, Redmond, Washington, USA). Statistical analyses were generated using SPSS for Windows V.15.0 (SPSS Inc, Chicago, Illinois, USA). Reporting of this study was done in accordance with the CONSORT statement. ${ }^{15}$ Statistical significance was taken as $\mathrm{p}<0.05$.

\section{RESULTS}

We enrolled 184 neurosurgical patients between April 2004 and December 2008. An interim report was presented during the ICP in 2007 in San Francisco, USA. ${ }^{15}$ Common barriers to recruitment included next of kin not available for consent before emergency neurosurgical operations or ongoing sepsis (suspected or confirmed). The trial patient profile is depicted in figure 1. Baseline characteristics are shown in table 1. There were no significant imbalances between the two groups. Ventricular

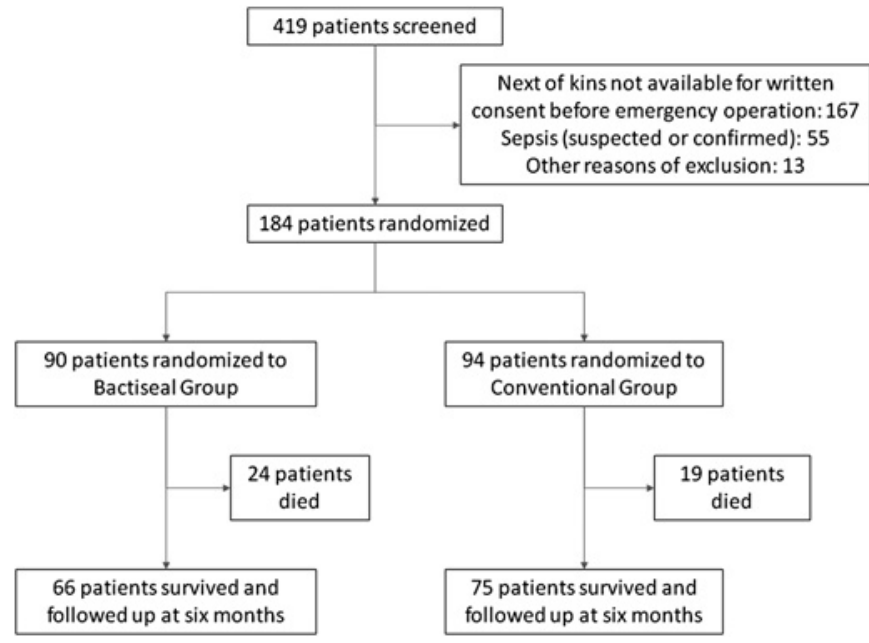

Figure 1 Trial profile.

catheter duration was 9.6 4.9 days for the Bactiseal group and $9.6 \pm 4.6$ days for the conventional group $(p=0.859)$.

In the primary outcome analysis, the proportion of patients with nosocomial infection was not significantly different $57 \%$ (51/90) in the Bactiseal group and 51\% (48/94) in the conventional group) (OR 1.3, CI 0.7 to 2.2). There were also no differences in the secondary outcome measures between the two groups. The proportion of patients with CNS infections as a function of the length of time the catheter remained in place was compared between the two groups using Kaplan-Meier curves (figure 2). There were four CSF infections, 1\% (1/90) in the Bactiseal group and $3 \%(3 / 94)$ in the conventional group ( $p$ $(\log$ rank $)=0.282$ ). The proportions of patients with a favourable outcome at 6 months were also not significantly different $37 \%$ $(33 / 90)$ in the Bactiseal group and $47 \%$ (44/94) in the conventional group) (OR 0.7, CI 0.4 to 1.2). ICU stay and acute hospital

Table 1 Patient characteristics

\begin{tabular}{|c|c|c|c|}
\hline & Bactiseal group & Conventional group & p Value \\
\hline Age (years) & & & 0.258 \\
\hline Mean (SD) & $53(14)$ & $51(14)$ & \\
\hline Median (range) & $55(20-82)$ & $51(18-83)$ & \\
\hline Sex & & & 0.958 \\
\hline Male & $53(59)$ & $55(59)$ & \\
\hline Female & $37(41)$ & $39(41)$ & \\
\hline Admission GCS & & & 0.863 \\
\hline Mean (SD) & $9(4)$ & $9(3)$ & \\
\hline Median (range) & $9(3-15)$ & $9(3-15)$ & \\
\hline ICP catheter duration & & & 0.859 \\
\hline Mean (SD) & $10(5)$ & $10(5)$ & \\
\hline Median (range) & $9(1-31)$ & $9(2-27)$ & \\
\hline Neuropathology & & & 0.801 \\
\hline Stroke & $61(68)$ & $67(71)$ & \\
\hline Head Injury & $20(22)$ & $20(21)$ & \\
\hline Other & $9(10)$ & $7(7)$ & \\
\hline \multicolumn{4}{|l|}{ Known risk factor } \\
\hline Craniotomy & $57(63)$ & $57(61)$ & 0.707 \\
\hline CSF leakage from wound & 7 (8) & $6(6)$ & 0.712 \\
\hline Skull base fracture & $11(12)$ & $12(13)$ & 0.911 \\
\hline Open skull vault fracture & $3(3)$ & $0(0)$ & 0.115 \\
\hline Intraventricular haemorrhage & $30(33)$ & $35(94)$ & 0.581 \\
\hline Diabetes & $7(8)$ & $9(10)$ & 0.665 \\
\hline Steroid treatment & $6(7)$ & $6(6)$ & 0.938 \\
\hline
\end{tabular}

Data are numbers (\%), unless otherwise indicated.

GCS, Glasgow Coma Scale; ICP, intracranial pressure. 
Kaplan Meier survival estimates, Bactiseal group versus conventional catheter group

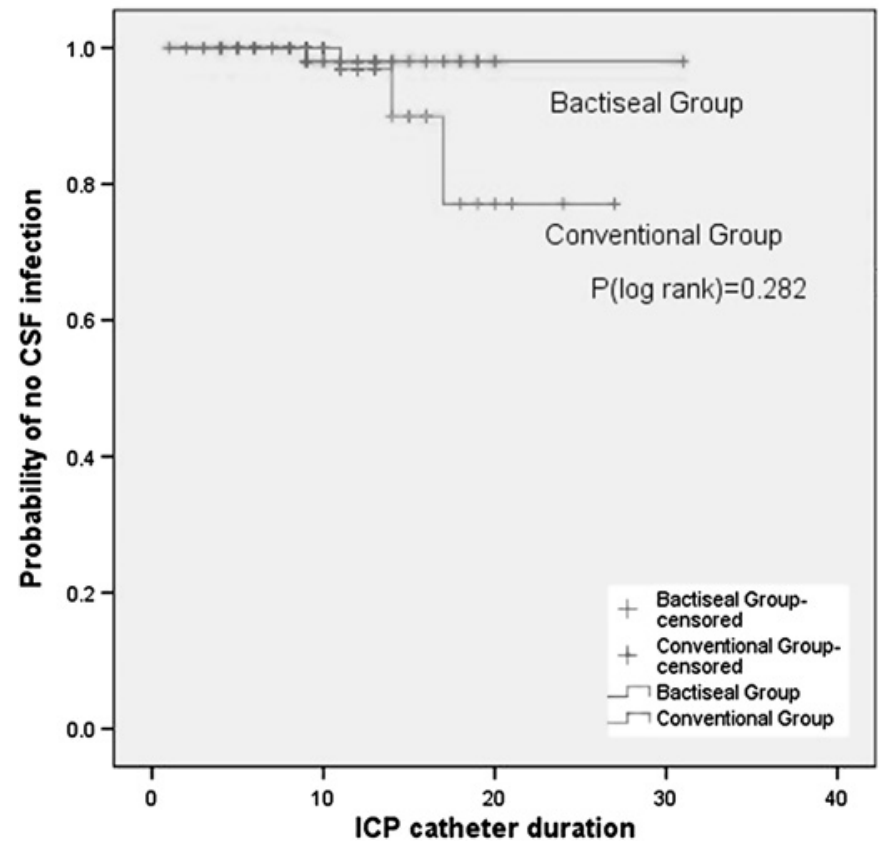

Figure 2 CSF infection versus days of intracranial pressure (ICP) monitoring catheters.

stay were also not significantly different between the two groups. ICU stay was $6.9 \pm 5.5$ days for the Bactiseal group and $7.3 \pm 5.5$ days for the conventional group $(p=0.520)$. Acute hospital stay was $26.2 \pm 19.8$ days for the Bactiseal group and $25.2 \pm 18.6$ days for the conventional group ( $p=0.997)$.

\section{Infection and microbiology profile}

There were four CNS infections, with one in the Bactiseal group and three in the conventional group. Organisms responsible included coagulase negative staphylococci $(n=1)$, staphylococci epidermidis $(n=1)$, Acinetobacter $(n=1)$ and Serratia $(n=1)$. All were managed with 2-4 weeks of intravenous antibiotics and removal/exchange of ventricular catheters. At 6 months, only one $(25 \%)$ achieved a favourable neurological outcome. Ventricular catheter ends (about $5 \mathrm{~cm}$ in length) were saved for culture. There were seven positive cultures of ventricular catheter tips without evidence of CSF infection. Two were in the Bactiseal group and five were in the conventional group. Organisms cultured included coagulase negative staphylococci $(\mathrm{n}=4)$, methicillin resistant Staphylococcus aureus (MRSA) $(\mathrm{n}=1)$, diphtheroids $(\mathrm{n}=1)$ and Acinetobacter $(\mathrm{n}=1)$.

There were three cases of pseudomembranous colitis from the conventional group and none from the Bactiseal group $(p=0.246)$. One patient required total colectomy to treat colitis.

There were also no significant differences in different nonCNS infection rates between the Bactiseal and conventional groups. Chest infections occurred in 43/90 (48\%) patients in the Bactiseal group and in $34 / 94(36 \%)$ in the conventional group (OR 1.6, CI 0.9 to 2.9). Urinary tract infection occurred in $20 / 90$ $(22 \%)$ patients in the Bactiseal group and in $11 / 94(12 \%)$ in the conventional group (OR 2.2, CI 0.97 to 4.8). Septicaemia occurred in 6/90 (7\%) patients in the Bactiseal group and in 8/94 $(9 \%)$ in the conventional group (OR 0.8 , CI 0.3 to 2.3 ).

In terms of microbiological profile, we compared incidences of resistant infections related to MRSA, Acinetobacter, fungi,
Pseudomonas and community onset extended spectrum $\beta$-lactamase (ESBL) producing Escherichia coli between the Bactiseal and conventional groups. Overall, resistant infections occurred in $27 / 90(30 \%)$ patients in the Bactiseal group and in $34 / 94$ $(36 \%)$ in the conventional group (OR 0.8 , CI 0.4 to 1.4 ). There were also no significant differences in the named resistant infections between the two groups. MRSA infection was present $2 / 90$ (2\%) in the Bactiseal group and in 4/94 (4\%) in the conventional group (OR 0.5, CI 0.1 to 2.9). Acinetobacter infection occurred in $7 / 90$ (8\%) patients in of the Bactiseal group and in 13/94 (14\%) in the conventional group (OR 0.5 , CI 0.2 to 1.4). Fungal infection occurred in $7 / 90$ (8\%) patients in the Bactiseal group and in 12/94 (13\%) in the conventional group (OR 0.6, CI 0.2 to 1.5). Pseudomonas infection occurred in $12 / 90(13 \%)$ patients in the Bactiseal group and in $18 / 94$ $(19 \%)$ in the conventional group (OR 0.7 , CI 0.3 to 1.4$)$. ESBL producing $E$ coli infection occurred in $6 / 90$ (7\%) patients in the Bactiseal group and in $3 / 94(3 \%)$ in the conventional group (OR 2.1, CI 0.5 to 8.9 ).

Patients with ventricular catheters in situ for more than $\mathbf{5}$ days A total of 154 (84\%) patients had a ventricular catheter in situ for more than 5 days. Seventy-four patients were in the Bactiseal group and 80 were in the conventional group. Again, there were no differences in CSF infection rates, nosocomial infection rates or incidences of infections related to MRSA, Acinetobacter, fungi, Pseudomonas or ESBL producing E coli between the Bactiseal and conventional groups. The proportion of patients with nosocomial infection was $64 \%(47 / 74)$ in the Bactiseal group and $56 \%$ $(45 / 80)$ in the conventional group (OR 1.4 , CI 0.7 to 2.6).

\section{Clinical safety}

Clinical safety was evaluated by comparing the frequency of each complication (other than CSF infection) between the Bactiseal and conventional groups. The two complications noted were blocked catheter requiring another operative procedure for exchange and intracranial haemorrhage related to catheter insertion. There were no significant differences in the rate of reported complications in comparing the Bactiseal and conventional groups. Blocked catheter requiring exchange occurred in 6/ $90(7 \%)$ patients in the Bactiseal group and in 5/94 (5\%) in the conventional group (OR 1.3, CI 0.4 to 4.3). Intracranial haemorrhage related to catheter insertion occurred in $2 / 90(2 \%)$ patients in the Bactiseal group and in $1 / 94$ (1\%) in the conventional group (OR 2.1, CI 0.2 to 23.7).

\section{DISCUSSION}

This study was designed to assess the effectiveness of antibiotic impregnation compared with systemic antibiotics for patients requiring external ventricular drain insertion. We found that there was no difference in CSF infection and nosocomial infection rates between both groups. Resistant opportunistic infection rate (MRSA, Acinetobacter, fungi, Pseudomonas and ESBL producing $E$ coli) were similar between the two groups groups. We thus conclude that antibiotic impregnated catheters are as effective as systemic antibiotics.

Various experimental models had shown that antibiotic impregnated CSF shunt infection could prevent colonisation and did not increase the risk of postoperative seizures. ${ }^{8}{ }^{15-18}$ Two trials showed the benefit of antibiotic impregnated catheters in terms of CSF infection prevention. ${ }^{9}{ }^{19}$ Compared with ventriculoperitoneal shunt, ${ }^{20}$ use of antibiotics for prevention of CSF infection in patients with external ventricular catheter had been controversial. $^{21}$ Some authors did not report any benefit for 
prophylactic antibiotics. ${ }^{42-24}$ Our use of broad antibiotic cover was based on the results of two previous randomised controlled clinical trials carried out in our unit. ${ }^{10} 11$ Resistant opportunistic infections are always a concern with the use of broad spectrum antibiotic prophylaxis. The study result suggests that any theoretical increase in the risk of opportunistic infection is balanced out by prevention of concomitant infection. With the availability of antibiotic impregnated catheters, which is equivalent to local antibiotic prophylaxis, they should theoretically have the same capacity for CSF infection prevention, with the possibility of avoiding secondary resistant nosocomial infections. The latter is of particular interest, which forms an argument for use of antibiotic impregnated catheters. In terms of cost calculations, the daily cost of prophylactic antibiotics is HK\$466 (US\$60) whereas the cost of Bactiseal catheter locally is $\mathrm{HK} \$ 2700$ (US\$346) in excess of the cost of plain ventricular catheter. It is actually more cost effective if the ventricular catheter is required for more than 6 days, which is shorter than the means of 10 days of ventricular catheter in situ in the current study. However, we could not show any reduction in resistant infections from MRSA, Acinetobacter, fungi, Pseudomonas or ESBL producing $E$ coli with the use of antibiotic impregnated catheters, probably related to the smaller than expected observed difference (6\%). ${ }^{13}$ We also showed that even though theoretically antibiotic impregnation might make the catheter stiffer, there was no increase in catheter related complications such as intracranial haemorrhage.

The weakness of the current study included the unblinded nature of the available study catheters, and that the sample size was not powered to detect a smaller difference in infection rates. There is a recent report that antibiotic impregnation may increase false negative culture. ${ }^{25}$ Nevertheless, without additional systemic antibiotic treatment, they should all be detected eventually by positive cultures. With a background protocol of dual antibiotic prophylaxis in our institute, we were in a unique position to compare board spectrum prophylactic antibiotics with antibiotic impregnated catheters. We were able to confirm the effectiveness of antibiotic impregnated catheters in terms of prevention of CSF infection, with a lower overall cost compared with prophylactic antibiotics. Moreover, the study provided invaluable data for the design of a study focusing on nosocomial infection prevention in this group of neurosurgical patients.

\begin{abstract}
Acknowledgements We thank the research nurses Louise Wong for implementation of the study and Michael Lo for coordinating the trial documents. We would especially like to thank the patients and their relatives who agreed to participate in the study and all the medical and nursing staff who have supported the trial.
\end{abstract}

Funding The study was supported in part by Codman, Johnson and Johnson, USA. The funding sources had no role in the study design, data collection, data analysis, data interpretation or writing of the report. The corresponding author had full access to all of the data in the study and had final responsibility for the decision to submit for publication.

Competing interests None.

Patient consent Obtained

Ethics approval This study was conducted with the approval of the joint CUHK/NTEC ethics committees.

Contributors GKCW and WSP conceived and designed the study. MIP was responsible for the microbiological data. RYTN, CWKM and GKCW were responsible for patient recruitment. GKCW performed the statistical analysis and drafted the manuscript. All authors reviewed, amended and agreed on the final version of the manuscript.
Provenance and peer review Not commissioned; externally peer reviewed.

\section{REFERENCES}

1. Hoefnagel D, Dammers R, Ter Laak-Poort MP, et al. Risk factors for infections related to external ventricular drain. Acta Neurochir (Wien) 2008;150:209-14.

2. Holloway KL, Barnes T, Choi S, et al. Ventriculostomy infections: the effects of monitoring duration and catheter exchange in 584 patients. J Neurosurg 1996;85:419-26.

3. Paramore CG, Turner DA. Relative risks of ventriculostomy infection and morbidity Acta Neurochir (Wien) 1994:127:79-84.

4. Rebuck JA, Murry KR, Rhoney DH, et al. Infection related to intracranial pressure monitors in adults: analysis of risk factors and antibiotic prophylaxis. J Neurol Neurosurg Psychiatry 2000;69:381-4.

5. Rosner MJ, Becker DP. ICP monitoring: complications and associated factors. Clin Neurosurg 1976:23:494-519.

6. Rossi S, Buzzi F, Paparella A, et al. Complications and safety associated with ICP monitoring: a study of 542 patients. Acta Neurochir Suppl (Wien) 1998; 71:91-3.

7. Winfield JA, Rosenthal P, Kanter RK, et al. Duration of intracranial pressure monitoring does not predict daily risk of infective complications. Neurosurgery 1993; 33:424-31

8. Bayston R, Lambert E. Duration of protective activities of cerebrospinal fluid infection shunt catheters impregnated with antimicrobial agents to prevent bacterial catheter-related infection. J Neurosurg 1997;87:247-51.

9. Zabramski JM, Whiting D, Darouiche RO, et al. Efficacy of antimicrobialimpregnated external ventricular drain catheters: a prospective, randomized, controlled trial. J Neurosurg 2003;98:725-30.

10. Poon WS, Ng S, Wai S. CSF antibiotic prophylaxis for neurosurgical patients with ventriculostomy: a randomized study. Acta Neurochir Suppl (Wien) 1998; 71:146-8

11. Wong GK, Poon WS, Lyon D, et al. Cefepime vs. ampicillin/sulbactam and aztrenam as antibiotic prophylaxis in neurosurgical patients with external ventricular drain: result of a prospective randomized controlled clinical trial. J Clin Pharm Ther 2006;31:231-5.

12. Wong GK, Poon WS, Wai $S$, et al. Failure of regular external ventricular drain exchange to reduce cerebrospinal fluid infection: result of a randomized controlled trial. J Neurol Neurosurg Psychiatry 2002:73:759-61.

13. Casagrande JT, Pike MC, Smith PG. An improved approximate formula for calculating sample sizes for comparing two binomial distributions. Biometrics 1978;34:483-6.

14. Wilson JTL, Pettigrew LEL, Teasdale GM. Structured interviews for the Glasgow outcome scale and extended Glasgow outcome scale: guidelines for their use. J Neurotrauma 1998:15:573-85.

15. Moher D, Schulz KF, Altman D. The CONSORT statement: revised recommendations for improving the quality of reports of parallel group randomized clinical trials. JAMA 2001;285:1987-91.

16. Wong GK, Poon WS, Ng SC, et al. The impact of ventricular catheter impregnated with antimicrobial agents on infections in patients with ventricular catheter: interim report. Acta Neurochir Suppl (Wien) 2008;102:53-5.

17. Abed WT, Alavijeh MS, Bayston R, et al. An evaluation of the epileptogenic properties of a rifampicin/clindamycin-impregnated shunt catheter. Br J Neurosurg 1994;8:725-30.

18. Kochro RA, Hampl JA, Jansen B, et al. Use of scanning electron microscopy to investigate the prophylactic efficacy of rifampicin-impregnated CSF shunt catheters J Med Microbiol 2000;49:441-50.

19. Pattavilakom A, Kotasnas D, Korman TM, et al. Duration of in vivo antimicrobial activity of antibiotic-impregnated cerebrospinal fluid catheters. Neurosurgery 2006; 58:930-5.

20. Secer HI, Kural C, Kaplan M, et al. Comparison of the efficacies of antibiotic impregnated and silver-impregnated ventricular catheters on the prevention of infections. An in vitro laboratory study. Pediatr Neurosurg 2008;44:444-7.

21. Harrop JS, Maltenfort MG, Ratliff JK, et al. Are ventriculostomy infections reduced by standardized protocols or by antibiotic-impregnated catheters? Neurosurgery 2009:65:405

22. Ratilal B, Costa J, Sampaio C. Antiobiotic prophylaxis for surgical introduction of intracranial ventricular shunts. Cochrane Database Syst Rev 2006;3:CD005365

23. Lozier AP, Sciacca RR, Romagnoli MF, et al. Ventriculostomy-related infections: A critical review of the literature. Neurosurgery 2002;51:170-82.

24. Alleyne CH, Hassan M, Zabramski JM. The efficacy and cost of prophylactic and periprocedural antibiotics in patients with external ventricular drains. Neurosurgery 2000:47:1124-9

25. Stevens EA, Palavecino E, Sherertz RJ, et al. Effects of antibiotic-impregnated external ventricular drains on bacterial culture results: an in vitro analysis. $J$ Neurosurg 2009 doi: 10.3171/2009.10.JNS091123. 